\title{
Analysis of Errors in Zero-Free-Parameter Modeling Approach to Predict the Voltage of Electrochemical Energy Storage systems under Arbitrary Load
}

\author{
Burak Ulgut*,Can Berk Uzundal and Elif Özdemir \\ Department of Chemistry, Bilkent University, 06800, Ankara, Turkey
}

\begin{abstract}
:
In a recently published article (J. Electrochem. Soc. 164 (2017) A1274-A1280), we described a new method to predict the voltage response of electrochemical energy storage systems during arbitrary load profiles. Our work shows that the impedance spectrum can be employed in the frequency domain in order to ultimately calculate the time domain behavior of the electrochemical energy storage system. The big advantage of this method is the fact that there are no free parameters and fits throughout.

The present work deals with the sources of error in the above-mentioned prediction approach and looks for the effects of the various sources of error. The current analysis concludes that two big contributors to the overall error are the inaccuracies in the DC part of the prediction and the non-linearities that are not modeled by a linear impedance spectrum.
\end{abstract}

Discussions are also made regarding ways to improve the performance of the modeling approach the most and where future work is going to be looking to improve.

\section{Introduction:}

Predictive modeling of battery response is of great interest to researchers and engineers in widely varying fields from automotive to consumer electronics to aviation [1-8]. Predicting the voltage of the battery throughout a discharge profile is of utmost importance to the design of any electrical powered product in order to pick and choose the right energy storage system.
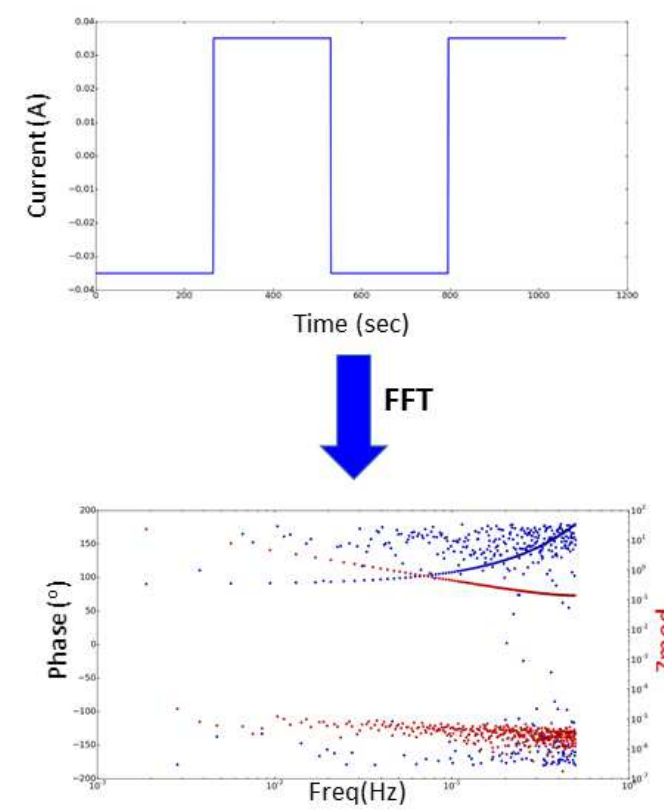
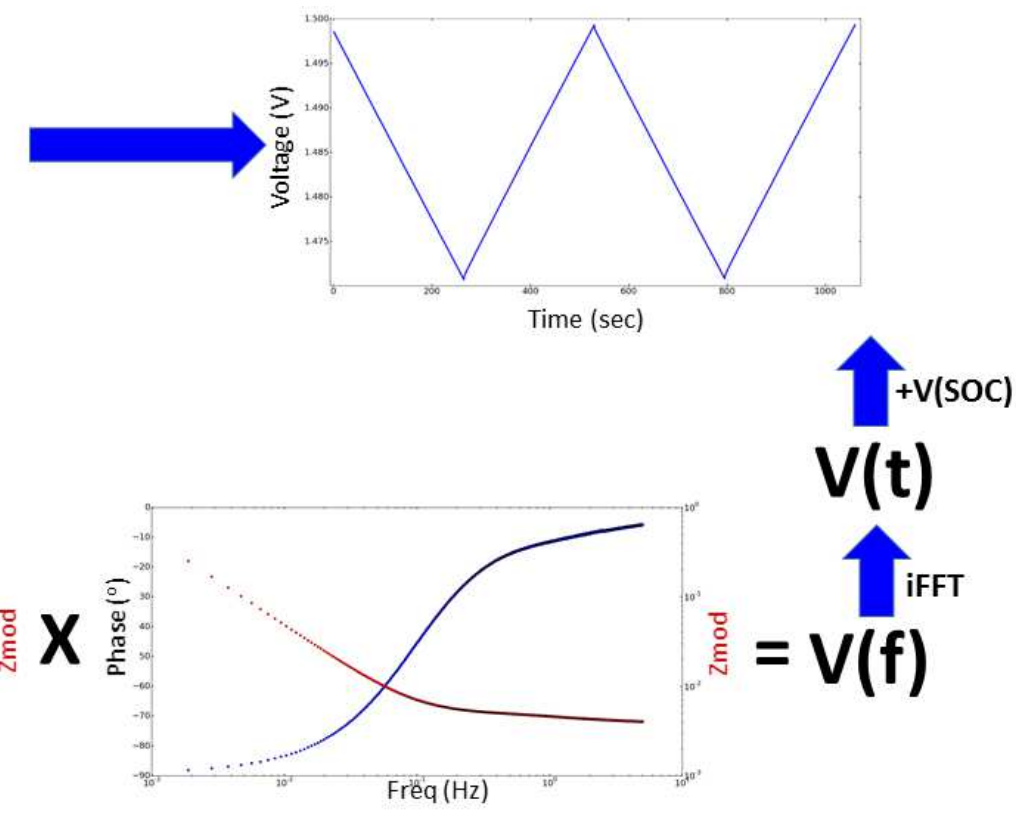

Figure 1 Overall summary of the approach. The current profile is Fourier transformed into the frequency domain and then multiplied with the impedance to obtain a frequency domain voltage. This voltage is then transformed back into the time domain. Finally, the DC behavior of the battery is added using a simple state-of-charge to DC voltage map.

In the literature, approaches to this problem can be widely grouped into two: equivalent circuit based models and first principle based models. Though both groups of models have been employed with varying success, both approaches suffer from drawbacks. The first principle models require the knowledge of numerous parameters regarding the kinetics and transport of various materials inside the system, most of which are not 
exactly known. This issue turns the problem into one of fitting with numerous parameters, which ends up over defining the problem. With the high degree of free parameters, the number of degenerate solutions increase greatly, in turn, decreasing the accuracy of the fit parameters obtained, precluding any reliable quantitative analysis.

The other approach to the problem, broadly described, is to use equivalent circuit modeling and then using the equivalent circuit models in time domain through a SPICE type approach [2,4]. In this approach, the components used in the impedance fitting are converted into a time domain analog (e.g. $[9,10])$ in order to model the time domain response of the system.

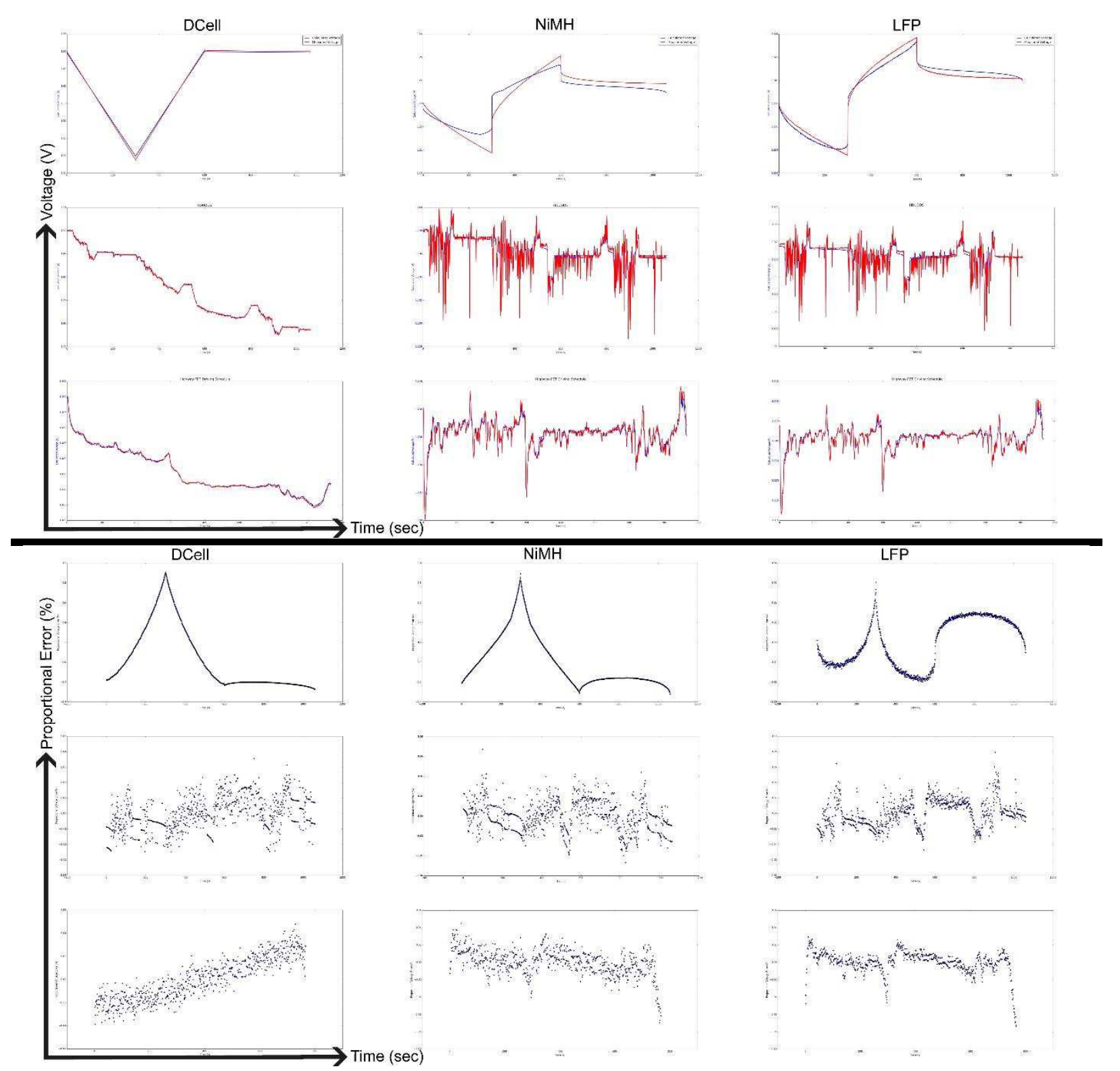

Figure 2The calculated results (top) and the fractional error(bottom) of the modeling approach shown. The fractional errors show non-random structure, especially in the squarewave signal.The columns show the three energy storage systems(350F, the NiMH and the $\mathrm{LiFePO}_{4}$, where the rows show three different discharge profiles, squarewave, HDUDDS from EPA and Highway FET from EPA.(For details, please see ref.9)

In the equivalent circuit based approaches to the problem, it is possible to define the circuits in a way to make sure that the number of degenerate solutions are minimized. However, these simplified circuit models (Simplified Randles and derivatives) fail to capture the full detail of the impedance spectrum [8]. There are models developed in the literature that do capture the fine detail in the impedance spectrum, however, these models have to take into account the porous structure of the electrodes and are generally based on transmission 
lines. These models are defined in the frequency domain and they are very complicated to get converted into the time domain.

Recently, a new method was reported by our group [9] that overcomes both these problems by using the impedance spectrum directly without any assumptions or fits. In short, this method employs the raw impedance data in the frequency domain without any attempt to fit or extract information from it. Instead, the discharge profile is transformed into the frequency domain and gets multiplied with the impedance measured at the relevant frequencies. In the end, the voltage in the time domain gets calculated through an inverse Fourier Transform followed by a correction for the DC voltage change due to the changes in the state of charge. This method is outlined in Fig. 1

The developed method has been successful in predicting the response of batteries and supercapacitors across a wide variety of discharge profiles. As we have described in the previous publication, this method is a zero-free-parameter method with straightforward algebra that clearly can predict the voltage response of the electrochemical energy storage system. Figure 2(top) shows the results obtained from the prediction algorithm. The columns are grouped with different energy storage systems whereas the rows show the three different discharge profiles used.

However, like in any prediction algorithm, the structure of

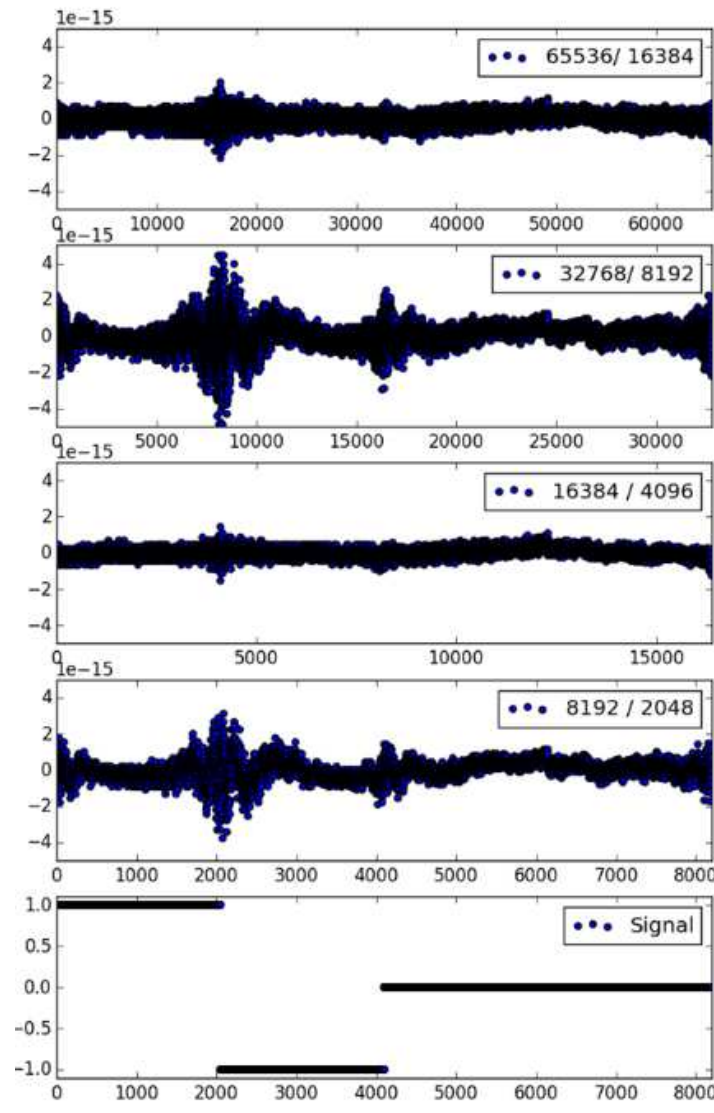

Figure 3 The results of the numerical test showing that the errors are not improving with improved sampling and that the error magnitude is too small to account for the observed errors.

the errors are worth investigating to not only decrease the errors, but also develop an understanding of the underlying phenomenon deeper. The present contribution is an attempt at investigating the errors and the contributing factors to the said errors.

\section{Error Analysis}

One way to investigate the success of the model is to look at the residuals [11]. The residuals in any fitting or prediction algorithm show if the errors seen are random or systematic. The observation of any systemic structure in the residuals indicate a fundamental part that is missing from the algorithm. Residuals for the model developed across different energy storage systems and the different discharge profiles are shown in Figure 2 bottom. The fact that the residuals are not random and that there is structure in the residuals indicates that a systematic error exists
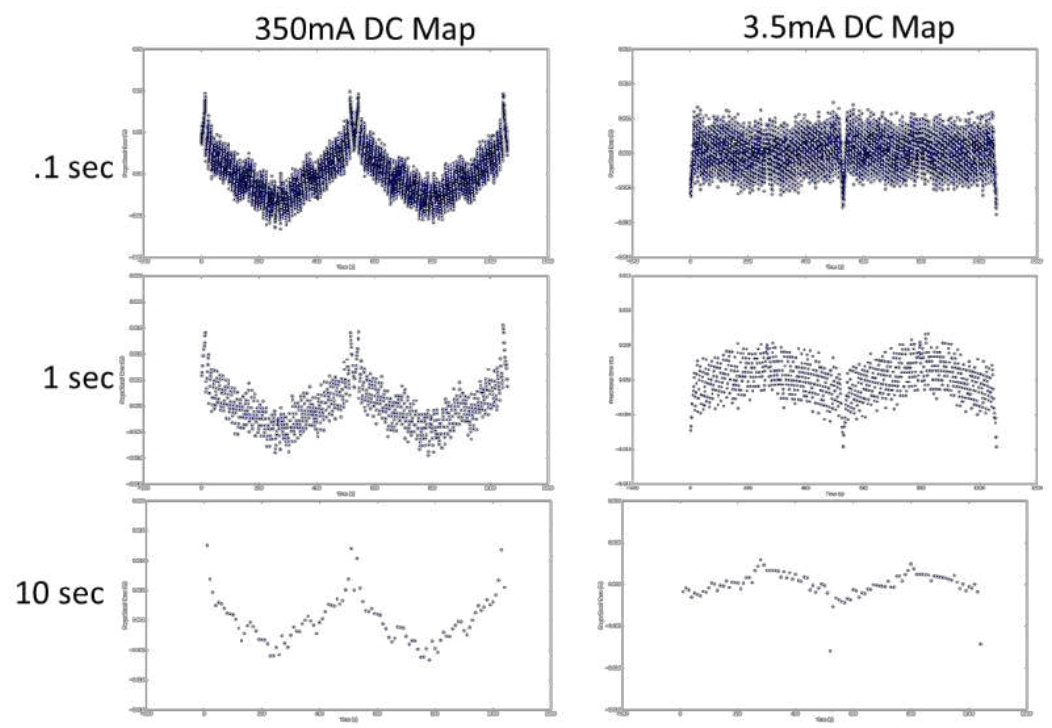

Figure 4 Experimental verification that changing the sampling rate does not affect the magnitude, nor the structure of the observed errors. in the model that is developed. Furthermore, the errors visually display more structure in regions where the discharge is stationary.

The existence of increased structure before the parts with sharp changes in current led us to believe that the reason for the systematic error might be introduced due to the approximation of the Fourier transform through 
a discrete Fourier Transform ([12,13]). This approximation is necessary for any sampled real system, but the validity is questionable under certain conditions. In order to validate our methodology, we have performed a numerical test and an experimental check.

\section{Experimental}

In the interest of clarity and focus, the experiments presented in this manuscript will be confined to a Squarewave and 350F supercapacitor. The Squarewave is defined using 1060 secs long sequence which has two periods of current signal in it. The amplitude and the sampling rate of the Squarewave are going to be varied in order to investigate the effects of the signal parameters to the error structure. Furthermore, the effects of the details of the DC Voltage vs. SOC map were investigated by measuring the DC map using different current values. The supercapacitor used is the Maxwell BCAP0350 E270 T11.

In order to investigate the errors without any bias from the measured voltage level, the errors were defined in a fractional manner, using the below equation.

$$
\text { Fractional Error }(t)=\frac{\text { Measured Voltage }(t)-\text { Calculated Voltage }(t)}{\text { Measured Voltage }(t)}
$$

The fractional errors as functions of time were investigated visually for signs of structure and amplitude changes as functions of various effects on the experimental parameters.

For the numerical check of the accuracy of a discrete Fourier Transform, the procedure was as follows. A signal was defined using the Pulse sequence that was used in [9]. The sampling rate for the signal was varied without changing the overall structure of the signal. Then, a Fourier Transform and an inverse Fourier Transform was performed. The experimental side of this check was performed using the below signal.

Further, we investigated the structure in the error experimentally through varying parameters regarding the signal amplitude, the current used for the DC map data and others in order to interrogate the main reason for the observed error structure. This error structure was evaluated visually without any further mathematical treatment.

For the experimental check, a square wave sampling frequency was varied from $0.1 \mathrm{sec}$ to $10 \mathrm{sec}$ in factors of 10 and the signal length was kept constant. The investigation was done for two different current amplitudes (350mA and $3.5 \mathrm{~mA}$ ). The amplitude of the signal was varied from $3.5 \mathrm{~mA}$ to $35 \mathrm{~mA}$ to $350 \mathrm{~mA}$ for different sampling rates. The DC map was collected with 3 different DC current amplitudes (from $3.5 \mathrm{~mA}$ to $350 \mathrm{~mA}$ changing in factors of 10). These amplitudes was chosen to vary over three orders of magnitude and their effects to the success of the prediction algorithm were investigated.

\section{Results \& Discussion}

The difference between the resulting data and the original data in the numerical test described above is plotted in Figure 3 in a normalized fashion. The results clearly indicate that first, the increased sampling rate doesn't change the errors significantly and second, the overall errors neither have the structure, nor the amplitude to explain the errors that was observed in the results. The exact nature of the error structure in this numerical experiment is beyond the scope of this

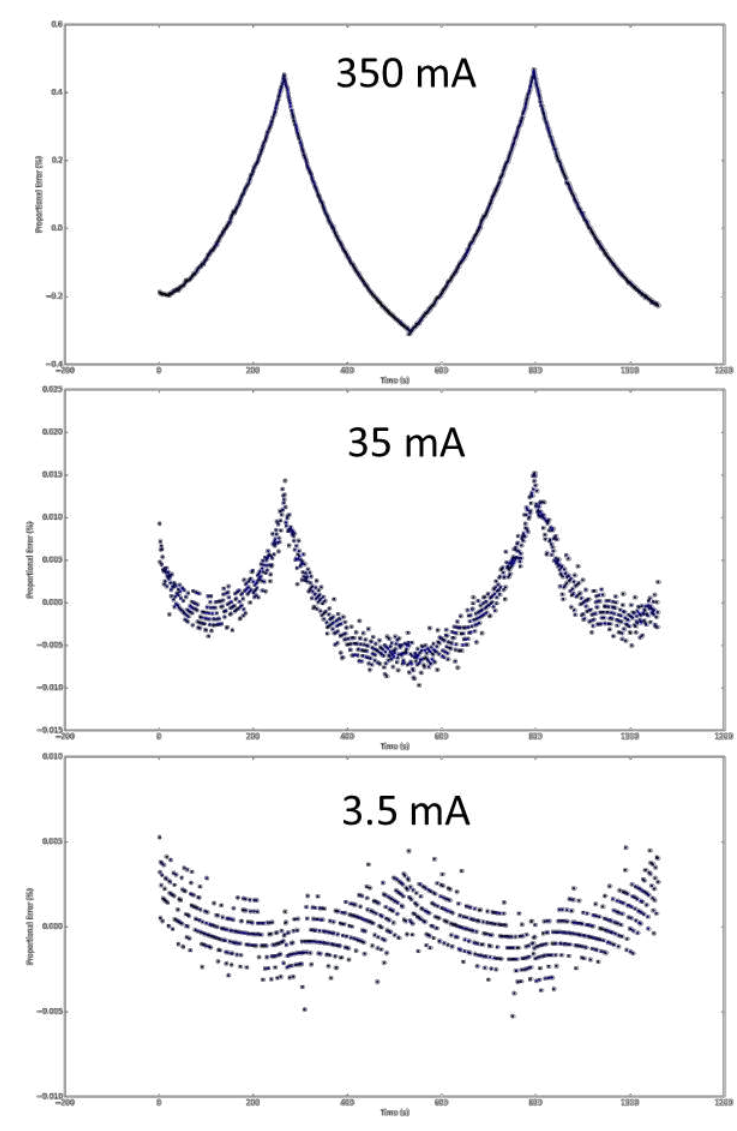

Figure 5 The observed errors at different current amplitudes. At higher amplitudes, the errors go higher since the nonlinearities get worse with increasing amplitudes 
study, however, it is curious to observe that the errors seem the worst in regions of the curve where the derivative is undefined.

The sampling issue was also checked experimentally where the same signal was used to measure the response with 0.1 $\mathrm{sec}, 1.0 \mathrm{sec}$ and $10.0 \mathrm{sec}$ sampling times respectively with different DC maps and different current amplitudes. Across the two orders of magnitude that the sampling rate changed, the changes in the sampling rate do not seem to change the error structure, nor the amplitude by any measure as shown in Figure 4. For clarity, only two DC maps are shown, but this is reproducible across different DC maps and different amplitudes. This experiment clearly shows that the error structure, nor the amplitude is not a function of the sampling rate.

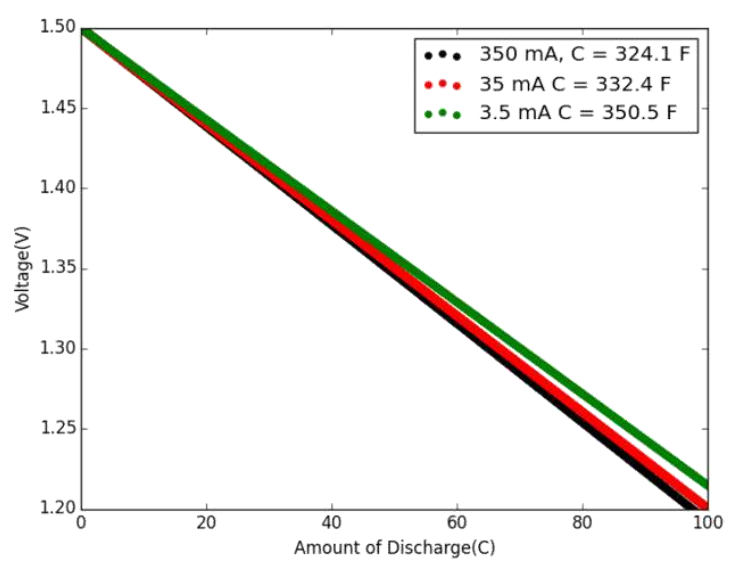

Figure 6 The cell voltage vs. amount of discharge map for the same 350F supercapacitor measured at different discharge rates. Lower currents result in higher apparent capacitance.

Both the numerical and the experimental evidence clearly show that within the range of parameters relevant to this system and the approach, the sampling rate is not a major factor in determining the amount nor the structure in the error.

In order to check for effects of linearity, the amplitude of the current pulse used was varied. As the amplitude gets lower, the nonlinearities should start decaying and the verifiably linear impedance measurement should start explaining the behavior of the system more accurately. As shown in Figure 5, as the current amplitude decreases, the error starts diminishing in amplitude and the overall structure starts to fade. More work is necessary in the modeling process to incorporate the non-linearities of the system into this approach.

The last effect we have checked is the effect of how the DC map is measured. The DC map is acquired with three different amplitudes, decreasing with factors of 10 from $350 \mathrm{~mA}$ to $3.5 \mathrm{~mA}$. The DC maps obtained are shown in figure 6. Using simple linear fits, the apparent capacitance of the sample can be calculated. The calculated capacitance for the 350F nameplate supercapacitor is $324.1 \mathrm{~F}$ for a DC map measured with $350 \mathrm{~mA}$, goes up to $332.4 \mathrm{~F}$ for $35 \mathrm{~mA}$ and $350.5 \mathrm{~F}$ for $3.5 \mathrm{~mA}$. The $350 \mathrm{~F}$ nameplate, as specified by the manufacturer specifies the capacitor using a 72 hour charging and has a precision of $+20 \% /-10 \%$.
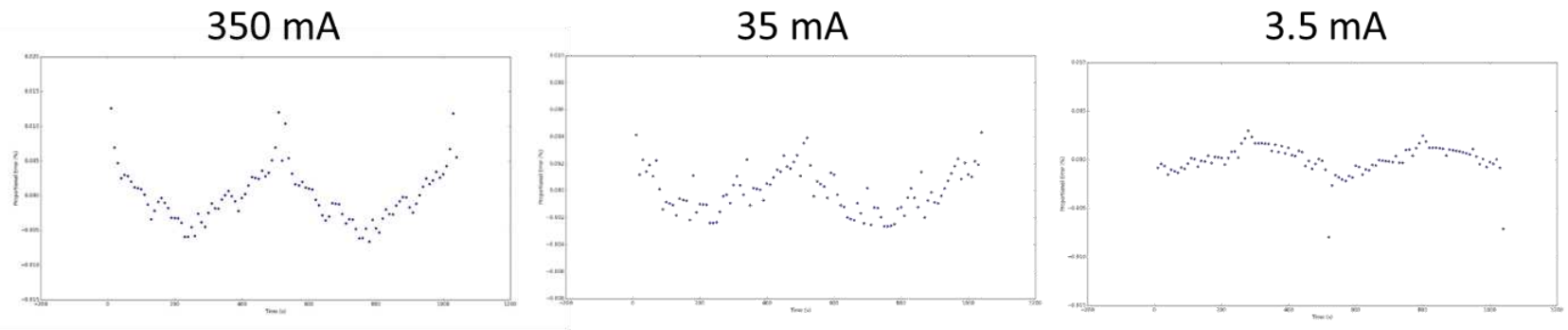

\section{$10 \mathrm{sec}, 3.5 \mathrm{~mA}$ Amplitude}
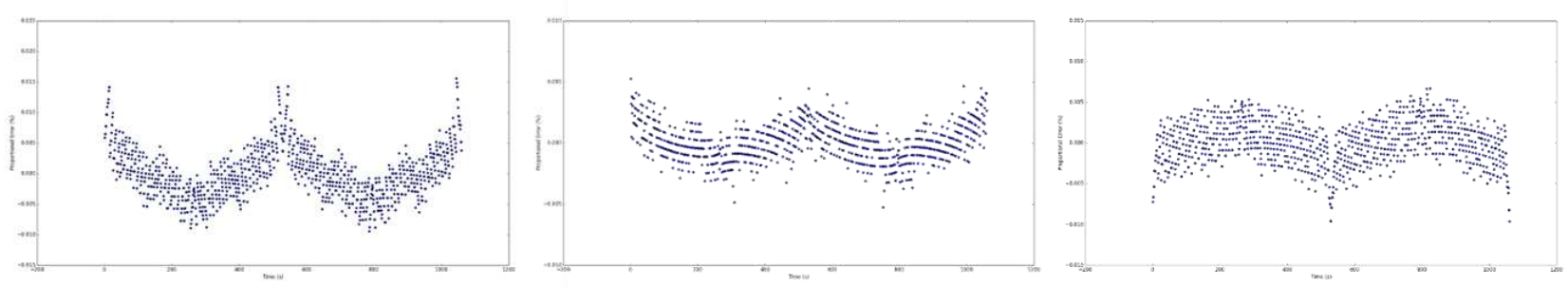

\section{$1 \mathrm{sec}, 3.5 \mathrm{~mA}$ Amplitude}

Figure 7 The effect of the quality of DC map is shown. The current values shown as column headings indicate the current that was used to collect the DC map data. 
Intuitively, the DC map is expected to be more accurate as the DC current used to collect the map goes down, which is shown to be experimentally true as shown in the above discussion. When the error is investigated as a function of the DC map used, the data gathered is shown on Figure 7. The magnitude of the error, in addition to the structure seem to decay with the decreasing current that was used to collect the DC map. This is a clear sign that at least part of the structure in the error is due to inaccuracies in defining the cell potential as a function of the state-of-charge.

\section{Conclusions and outlook.}

Based on the evaluations presented, the overall error magnitude and the structure seems to be dominated by the accuracy of the DC map in addition to the inaccuracies regarding linearity.

Further research will start looking into incorporating the higher harmonics of the EIS measurement. There is already theoretical work that is published regarding the investigations of higher harmonics [14], that we intend to build upon in the experimental side.

\section{References}

1. S. Nejad, D.T. Gladwin, D.A. Stone, A systematic review of lumped-parameter equivalent circuit models for real-time estimation of lithium-ion battery states, J. Power Sources. 316 (2016) 183-196. doi:10.1016/j.jpowsour.2016.03.042.

2. S. Castano, L. Gauchia, E. Voncila, J. Sanz, Dynamical modeling procedure of a Li-ion battery pack suitable for real-time applications, Energy Convers. Manag. 92 (2015) 396-405. doi:10.1016/j.enconman.2014.12.076.

3. T. Osaka, T. Momma, D. Mukoyama, H. Nara, Proposal of novel equivalent circuit for electrochemical impedance analysis of commercially available lithium ion battery, J. Power Sources. 205 (2012) $483-$ 486. doi:10.1016/j.jpowsour.2012.01.070.

4. E. Barsoukov, J.H. Kim, C.O. Yoon, H. Lee, Universal battery parameterization to yield a non-linear equivalent circuit valid for battery simulation at arbitrary load, J. Power Sources. 83 (1999) 61-70. doi:10.1016/S0378-7753(99)00257-8.

5. G. Fan, K. Pan, M. Canova, J. Marcicki, X.G. Yang, Modeling of Li-Ion Cells for Fast Simulation of High C-Rate and Low Temperature Operations, J. Electrochem. Soc. 163 (2016) A666-A676. doi:10.1149/2.0761605jes.

6. M. Verbrugge, D. Baker, X. Xiao, Formulation for the Treatment of Multiple Electrochemical Reactions and Associated Speciation for the Lithium-Silicon Electrode, J. Electrochem. Soc. 163 (2016) A262-A271. doi:10.1149/2.0581602jes.

7. M. Thele, O. Bohlen, D.U. Sauer, E. Karden, Development of a voltage-behavior model for NiMH batteries using an impedance-based modeling concept, J. Power Sources. 175 (2008) 635-643. doi:10.1016/j.jpowsour.2007.08.039.

8. M. Slocinski, Method for parameterization of impedance-based models with time domain data sets, Lect. Notes Impedance Spectrosc. 4 (2013) 3-16.

9. E. Özdemir, C. B. Uzundal, B. Ulgut, Zero-Free Parameter Approach to Predict the Voltage of Batteries of Different Chemistries and Supercapacitors under Arbitrary Load, J. Electrochem. Soc. 164 (2017) A1274-A1280

10. B. Ulgut, J. E. Grose, Y. Kiya, D. C. Ralph, H.D. Abruña, A new interpretation of electrochemical impedance spectroscopy to measure accurate doping levels for conducting polymers: Separating Faradaic and capacitive currents, Appl. Surf. Sci. 256(2009) 1304-1308

11. N. R. Draper, H. Smith, Applied Regression Analysis. Wiley Interscience (1998).

12. C.L. Epstein, How well does the finite fourier transform approximate the fourier transform?, Commun. Pure Appl. Math. 58 (2005) 1421-1435. doi:10.1002/cpa.20064.

13. L. Auslander, F.A. Grunbaum, The Fourier transform and the discrete Fourier transform, Inverse Probl. 5 (1989) 149-164. doi:10.1088/0266-5611/5/2/004.

14. M.M. Murbach and D. T. Schwartz, Extending Newman's Pseudo-Two-Dimensional Lithium-Ion Battery Impedance Simulation Approach to Include the Nonlinear Harmonic Response, J. Electrochem. Soc. 164(2017)E3311-E3320 